\title{
Avaliação da presença de práticas do Design for Environment (DfE) no desenvolvimento de produto de uma empresa da indústria química
}

\author{
Miriam Borchardta* Marcos Henrique Wendt ${ }^{\mathrm{b}}$, \\ Miguel Afonso Sellittoc, Giancarlo Medeiros Pereira ${ }^{\mathrm{d}}$ \\ a*miriamb@unisinos.br, UNISINOS, Brasil \\ bmarcos.wendt@artecola.com.br, ARTECOLA, Brasil \\ 'sellitto@unisinos.br, UNISINOS, Brasil \\ dgian@unisinos.br, UNISINOS, Brasil
}

\begin{abstract}
Resumo
Este artigo apresenta a avaliação da presença de práticas do Design for Environment (DfE) ou ecodesign no desenvolvimento de produtos de uma empresa da indústria química. Para atingir o objetivo proposto, o DfE foi desdobrado em sete construtos latentes, que foram ponderados por um método multicriterial. Os construtos foram desdobrados em itens de avaliação, que deram origem a um questionário categórico, empregado para investigar o quanto cada item está presente na empresa. Ao final, foi possível identificar o nível de presença de cada construto e a presença global do DfE na empresa. 0 resultado foi uma presença de $67 \%$ do máximo que se poderia esperar. Também foi possivel identificar a importância relativa de cada construto e as lacunas entre a importância e o nível de presença. Os construtos mais importantes foram Características do produto/processo e Resíduos. Este trabalho constribuiu para a identificação de prioridades quando da aplicação do DfE e para o estabelecimento de parâmetros referenciais acerca do desempenho em DfE a serem monitorados a longo prazo.
\end{abstract}

Palavras-chave

Design for Environment. Ecodesign. Desempenho ambiental. Avaliação de desempenho.

\section{Introdução}

Um dos objetivos do desenvolvimento sustentável é conciliar crescimento econômico com preservação do meio ambiente, trazendo melhorias em condições sociais de comunidades (KAZAZIAN, 2005). Em ambiente corporativo, desenvolvimento sustentável pode ser entendido como um conjunto articulado de ações que visam construir simultaneamente prosperidade material para a empresa, qualidade ambiental para a região e bem-estar social para as comunidades envolvidas. Em situações de sustentabilidade ambiental, as atividades humanas não interferem nos ciclos naturais resilientes do planeta e não reduzem o capital natural que será transmitido às gerações futuras (HARTSHORN, 2005). Tais conceituações integram o desenvolvimento econômico e social com seus reflexos nos fluxos naturais de materiais e energia. Da discussão, emerge que a sustentabilidade é um objetivo a atingir, não uma direção a seguir.

Observa-se que nem toda proposta de ação ambiental pode ser considerada sustentável se não contemplar outros aspectos, além dos usuais tratamentos ambientais, tais como redução de emissões e tratamento de efluentes.

Alinhado com o exposto, surge o conceito da Ecologia Industrial proposto por Jay Forrester, na 
década de 1960, baseado na teoria dos sistemas. A Ecologia Industrial considera que todos os resíduos e materiais devem ser continuamente reciclados dentro de um sistema fechado. Somente a energia solar ilimitada seria utilizada de forma dissipativa. A Ecologia Industrial reúne técnicas, tais como: prevenção da poluição, Produção Mais Limpa e Design for Environment (ou Design for the Environment) ou ecodesign, e cria uma nova agenda de reorganização de operações industriais, entendidas no seu contexto mais amplo de atividades econômicas, não restritas à produção de bens manufaturados. 0 espectro de alcance da Ecologia Industrial, ainda sem definição completa e consensual, parece ser vasto, estendendo-se desde a dimensão microeconômica, ligada a vantagens econômicas para as empresas geradas pela redução da geração de poluentes e aproveitamento de resíduos em outras unidades, até a dimensão macroeconômica, em que um novo paradigma econômico-ambiental é construído na direção das práticas de sustentabilidade (COSTA, 2002).

Assim como a Ecologia Industrial, o Design for Environment (DfE) ou ecodesign ainda se encontra em fase de estruturação e desenvolvimento. 0 DfE é uma técnica de projeto de produto na qual os objetivos usuais de projeto, tais como desempenho, confiabilidade e custo de manufatura são considerados junto com objetivos ambientais, tais como redução em danos ambientais, redução do uso de recursos naturais, incremento da eficiência energética e reciclagem de materiais (FIKSEL, 1996). Dessa forma, é possível conjugar as funções do produto ou serviço com aspectos relacionados à sustentabilidade ambiental, reduzindo, por sua vez, o impacto ambiental e aumentando a presença de produtos ecoeficientes em mercados já maduros (KARLSSON; LUTTROPP, 2006). Para Vercalsteren (2001), empresas podem considerar o DfE como um meio para preservar não apenas o ambiente, mas também a competitividade, pela potencial vantagem financeira, e a imagem pública junto ao mercado e sociedade, o que permite atingir novos mercados. Lindahl (2006) destaca que, apesar de crescente o número de publicações sobre DfE, poucos relatos efetivos de aplicação em manufatura podem ser observados.

Diversos requisitos para o DfE foram observados. Os principais deles referem-se a materiais, componentes, processos e características dos produtos, uso de energia, armazenagem e distribuição, embalagem e resíduos (WIMMER et al., 2005; LUTTROPP; LAGERSTED, 2006; FIKSEL, 1996). No entanto, até quanto se pesquisou na literatura, essas práticas ambientais, relacionadas ao DfE, têm formato genérico e de difícil enquadramento a métodos específicos de projeto de produto e processos industriais (BORCHARDT et al., 2009; KNIGHT; JENKINS, 2009).

Autores como Cerdan et al. (2009), Cabezas et al. (2005), Svensson et al. (2006) e Daub (2007) têm trabalhado no desenvolvimento de indicadores de desempenho associados ao DfE; destacam, entretanto, que não há consenso quanto a esse tópico. Apesar de frequentemente se verificar a presença do tema desempenho ambiental na literatura (SELLITTO; BORCHARDT; PEREIRA, 2010; ZOBEL et al., 2002; MEL0; PEGADO, 2006), até quanto se pesquisou, não foram observadas publicações que tratem de definir, modelar ou avaliar o desempenho de práticas assumidas como DfE. Adicionalmente, disponibilidade restrita de recursos frequentemente forçam empresas a responder a pressões ambientais de modo limitado, exigindo uma postura estratégica para maximizar resultados com mínimos recursos (LEE; KLASSEN, 2008). Nesse caso, um modelo que ajude a avaliar o atual desempenho e, por consequência, a priorizar ações de correção pode ser útil na formulação de uma estratégia de desenvolvimento de produto centrada em DfE.

Tal constatação remete aos seguintes questionamentos: como avaliar se um conjunto de práticas de projeto adotadas no desenvolvimento de produto está ou não contribuindo para o DfE? Em síntese: como avaliar o desempenho do DfE em uma dada empresa de uma indústria? Com a busca da resposta para a questão levantada pretende-se evitar que esforços em DfE resultem contraditórios ou ineficazes e orientar a organização a priorizar os recursos limitados em que os ganhos ambientais sejam mais significativos.

Considerando o exposto, o objetivo principal deste trabalho é avaliar o desempenho do DfE em uma empresa. Para que o objetivo principal seja atingido, os seguintes objetivos secundários foram estabelecidos: i) identificar construtos latentes que expliquem o DfE; ii) propor um modelo, suportado por método de decisão multicriterial, para avaliar a importância relativa de cada construto do DfE; iii) elaborar um instrumento de avaliação da presença ou grau de aplicação de cada construto do DfE; e iv) aplicar o método proposto em um caso e analisar os resultados. Foi usada modelagem como método de pesquisa e o método proposto foi aplicado em uma empresa da indústria química. 0 DfE foi explicado por um modelo matemático que o relacionou a construtos latentes.

Na sequência, apresentam-se o referencial teórico sobre DfE e medição e avaliação de desempenho. Depois, detalha-se o procedimento metodológico. Posteriormente, apresentam-se os resultados da pesquisa e discutem-se os achados. 


\section{Referencial teórico}

\subsection{Design for Environment ou ecodesign}

A ideia do DfE surgiu na década de 1990, quando a indústria eletrônica dos EUA procurava minimizar o impacto no meio ambiente decorrente de sua atividade. A Associação Americana de Eletrônica (American Electronics Association) formou uma força-tarefa para desenvolver projetos com preocupação ambiental e providenciar uma base conceitual que beneficiasse primeiramente os membros da associação. Desde então, o nivel de interesse pelo assunto cresceu e os termos ecodesign e Design for Environment passaram a ser mencionados em programas de gestão ambiental (BORCHARDT et al., 2008).

No DfE, o projetista seleciona e articula soluções de projeto segundo seu impacto no ciclo de vida do produto: fabricação, embalagem, uso, troca de peças e fim de vida. Avalia também a utilização do produto, pois este não é independente nem homogêneo e exige outros produtos e atores para a sua fabricação, o seu transporte e o seu uso, em uma abordagem transversal e multidisciplinar. Tingström e Karlsson (2006) salientaram a multidisciplinaridade do DfE, considerando que o desenvolvimento de um novo produto não é um processo linear e repetitivo, é complexo, pois interações inesperadas entre o produto e o meio podem surgir, requerendo o uso de modelos não lineares para o seu teste.

Além da preocupação ambiental, Luttropp e Lagerstedt (2006) destacam a importância do equilibrio entre custos ambientais e aspectos funcionais dos produtos. Karlsson e Luttropp (2006) salientam que o DfE inclui prioridades relativas à sustentabilidade humana no cenário dos negócios e seu principal objetivo é a redução do impacto ambiental. Em Borchardt et al. (2010) é apresentado um caso de aplicação do ecodesign que somente foi possivel a partir da constatação de que os ganhos auferidos seriam tanto relativos aos aspectos ambientais como econômicos.
A aplicação do DfE requer uma análise de sua viabilidade na organização e, consequentemente, de sua inserção na rotina de desenvolvimento de produtos. Vercalsteren (2001) sugere que a organização avalie fatores internos, externos e relacionados ao produto conforme indicado no Quadro 1.

Embora os fatores apresentados por Vercalsteren (2001) sejam decorrentes da análise da viabilidade do DfE em PMEs (pequenas e médias empresas), Reyes, Millet e Bissaud (2006) apresentam os mesmos fatores como resultado da pesquisa sobre elementos que sustentam a implementação do DfE em companhias francesas, independente do porte. Como principais obstáculos à implementação do DfE, são apontados por Reyes, Millet e Bissaud (2006): falta de comprometimento da alta gerência, resistência à mudança, ausência ou a não percepção dos benefícios econômicos e comerciais, pouca ou nenhuma pressão legal, falta de experiência da equipe (destaca-se que a esse item foi atribuida uma importância menor que aos demais). Os autores também sugerem a organização dos elementos viabilizadores do DfE em fatores internos e externos e associados aos aspectos tecnológicos das companhias.

Uma vez identificado o potencial de uma organização para aplicar o DfE, é necessário o entendimento dos elementos que o constituem. Para tanto, referências sobre DfE e sua aplicação em manufatura foram consultadas na elaboração deste artigo. Bhamra (2004) e Charter (1997) tratam do ecodesign como opção estratégica para o desenvolvimento de novos produtos e melhoria de desempenho ambiental. Beitz (1993) aponta diretrizes para o projeto de produtos voltado à reciclabilidade. Brezet (1997) relaciona DfE e inovação, destacando que o DfE não precisa ser apenas reativo ou corretivo, podendo incorporar novas funções aos produtos. Park e Tahara (2008) sugerem que, em um projeto de produto com base em ecodesign, os requisitos de qualidade e de satisfação do cliente sejam considerados do modo integrado aos requisitos ambientais.

Quadro 1. Análise da viabilidade da aplicação do DfE em uma organização.

\begin{tabular}{|c|c|c|}
\hline Fatores internos & Fatores externos & $\begin{array}{l}\text { Fatores relacionados } \\
\text { ao produto }\end{array}$ \\
\hline $\begin{array}{l}\text { - Motivação da companhia; } \\
\text { - Inovação: considera a habilidade e capacidade de influenciar } \\
\text { especificações do produto; } \\
\text { - Competitividade: considerar que uma empresa líder em seu } \\
\text { mercado tem mais chance de reconfigurar os produtos; } \\
\text { uma empresa pequena pode considerar o DfE como uma } \\
\text { oportunidade de incrementar a participação no mercado; } \\
\text { - Setor: considerar que se já existem iniciativas no setor, a } \\
\text { companhia pode aprender com estas experiências. }\end{array}$ & $\begin{array}{l}\text { - Regulação; } \\
\text { - Clientes e mercado: é necessário avaliar se o } \\
\text { mercado aceitará ou não os "produtos verdes"; } \\
\text { - Fornecedores: é essencial comprometimento } \\
\text { e parceira. }\end{array}$ & $\begin{array}{l}\text { - Avaliar o potencial de um } \\
\text { reprojeto baseado nos } \\
\text { requisitos ambientais. }\end{array}$ \\
\hline
\end{tabular}

Fonte: adaptado de Vercalsteren (2001). 
Os aspectos relativos ao DfE encontrados na literatura (FIKSEL, 1996; WIMMER et al., 2005; LUTTROPP; LAGERSTED, 2006) foram organizados no Quadro 2. Este quadro deu origem à estrutura arborescente e ao questionário empregados na pesquisa de campo.

Tingström e Karlsson (2006) chamam a atenção para a multidisciplinaridade do DfE e, para tanto, o efeito do produto no ambiente deve ser testado ou mensurado por meio de modelos. Os autores também sugerem que suas práticas ambientais, relacionadas aos construtos do DfE e as estratégias de execução, sejam mensuradas por sistemas de medição que consideram a complexidade do objeto. Tal observação confirma a importância da avaliação do desempenho do DfE e remete ao uso de um método de decisão multicriterial.

\subsection{Avaliação de grandezas intangíveis: presença do DfE}

Observou-se na literatura pesquisada que não há distinções claras entre os termos medição e avaliação de grandezas intangiveis. Para esse trabalho, considera-se o exposto em Sellitto, Borchardt e Pereira (2006): fala-se em avaliação quando esta partir de dados obtidos por julgamentos de variáveis categóricas e em medição quando os dados se originarem de contagem, cálculo ou medição de campo de variáveis quantitativas. Um exemplo de grandeza intangivel associada ao DfE e que tem sido avaliada e medida na literatura por meio de indicadores é o desempenho ambiental de uma operação industrial.
Zobel et al. (2002) avaliam o impacto ambiental sob a perspectiva gerencial, considerando o ciclo de vida dos produtos. Thoresen (1999) propõe uma metodologia para medir o desempenho ambiental. Ammenberg, Hjelm e Quotes (2002) usam a gestão de sistema ambiental para melhorar e medir o desempenho ambiental. Jiménez e Lorente (2001) sugerem que a abordagem tradicional de gerenciamento das operações baseada no custo, qualidade, tempo e serviço não é suficiente no atual momento; os autores argumentam que é essencial considerar o desempenho ambiental também como um objetivo operacional. Melo e Pegado (2006) citam, entre outras, algumas vantagens de se ter um modelo para medição formal de desempenho por indicadores quantitativos: i) síntese e comunicação da complexa informação exigida para a gestão de sistemas ambientais; ii) medição das lacunas, a diferença entre o desempenho atual e as metas de desempenho fixadas pela gestão; e iii) entre as lacunas, identificação de prioridades de ação. Pegado, Melo e Ramos (2001) observam dois problemas em sistemas de medição de desempenho: indicadores exclusivos são pouco úteis na análise do efeito ambiental de cadeias produtivas; e alguns sistemas de medição dão mais importância a indicadores de eficiência na gestão do que aos efeitos ambientais causados ou não evitados pela gestão. Kuhre (1998 apud MELO; PEGADO, 2006) afirma que a medição evolutiva do desempenho ambiental, por modelo de medição, permite melhorar os resultados da gestão ambiental, mesmo sem um sistema formal de gestão.

Sellitto, Borchardt e Pereira (2010) apresentaram um método completo para modelar e avaliar o desempenho ambiental em operações industriais; os

Quadro 2. Construtos do DfE.

\begin{tabular}{|c|c|}
\hline Construtos & Detalhamento \\
\hline $\begin{array}{l}\text { Materiais: escolha } \\
\text { e consumo }\end{array}$ & $\begin{array}{l}\text { Capacidade de: i) usar matéria-prima mais próxima de seu estado natural; ii) evitar misturas de materiais não compatíveis; } \\
\text { iii) eliminar o uso de substâncias tóxicas, perigosas e carcinogênicas; iv) não usar matérias-primas capazes de gerar } \\
\text { resíduos classe } 1 \text { - perigosos; v) usar materiais reciclados e/ou renováveis; vi) limitar as emissões atmosféricas originadas } \\
\text { pelo emprego de compostos orgânicos voláteis. }\end{array}$ \\
\hline $\begin{array}{l}\text { Componentes do } \\
\text { produto: seleção } \\
\text { e escolha }\end{array}$ & $\begin{array}{l}\text { Capacidade de: i) recuperar componentes ou usar componentes recuperados; ii) o acesso aos componentes; iii) identificar } \\
\text { materiais e componentes; iv) determinar o grau de reciclagem da cada material e componente. }\end{array}$ \\
\hline $\begin{array}{l}\text { Características do } \\
\text { produto/processo }\end{array}$ & $\begin{array}{l}\text { Capacidade de: i) elaborar produtos com formas mais simples e que reduza o uso ou consumo de matérias-primas; } \\
\text { ii) projetar produtos com maior vida útil; iii) projetar produtos multifuncionais; iv) realizar upgrade no produto; } \\
\text { v) desenvolver um produto com design respeitando as tendências mundiais. }\end{array}$ \\
\hline Uso de energia & $\begin{array}{l}\text { Capacidade de: i) usar energia proveniente de recursos renováveis; ii) empregar dispositivos de redução de consumo de } \\
\text { energia durante uso do produto; iii) reduzir consumo de energia durante a produção do produto; iv) reduzir consumo de } \\
\text { energia durante armazenamento do produto. }\end{array}$ \\
\hline $\begin{array}{l}\text { Distribuição de } \\
\text { produtos }\end{array}$ & $\begin{array}{l}\text { Capacidade de: i) planejar a logística de distribuição; ii) privilegiar fornecedores/distribuidores localizados mais próximos; } \\
\text { iii) minimizar os estoques; iv) usar modal de transporte mais eficiente em termos energéticos. }\end{array}$ \\
\hline $\begin{array}{l}\text { Embalagem e } \\
\text { documentação }\end{array}$ & $\begin{array}{l}\text { Capacidade de: i) reduzir peso e complexidade das embalagens; ii) usar documentação eletrônica; iii) usar embalagens que } \\
\text { possam ser reaproveitadas; iv) usar embalagens produzidas a partir de matérias-primas reaproveitadas; v) usar produtos } \\
\text { com refil. }\end{array}$ \\
\hline Resíduos & $\begin{array}{l}\text { Capacidade de: i) minimizar resíduos gerados no processo produtivo e no uso do produto; ii) reaproveitar os resíduos } \\
\text { gerados; iii) garantir limites aceitáveis de emissões; iv) eliminar a presença de resíduos classe } 1 \text { perigosos. }\end{array}$ \\
\hline
\end{tabular}

Fonte: adaptado de Wimmer et al. (2005); Luttropp e Lagerstedt (2006); Fiksel (1996). 
autores identificaram os construtos latentes capazes de compor o termo de topo desempenho ambiental. Foram premissas do método: i) o desempenho ambiental de uma operação antrópica pode ser desdobrado em construtos latentes que descrevem como as atividades da operação impactam o ambiente; ii) os construtos podem ser apreendidos por indicadores; iii) a prioridade dos construtos é variável e pode ser atribuída por gestores; e iv) os indicadores podem ser agregados formando um índice global que oscila entre 0\% e 100\%, para comunicação e comparação entre operações. 0 resultado final informa as condições instantâneas da operação, segundo as circunstâncias apreendidas pelo modelo, pode servir como série histórica e desempenhar a função de retroalimentação na tomada de decisões e na definição, avaliação e melhoria da estratégia ambiental da operação.

Para Bititci (1995), um sistema de medição ou de avaliação de desempenho deve: i) evitar a subotimização local; ii) desdobrar os objetivos estratégicos até os níveis operacionais; iii) proporcionar o pleno entendimento da estrutura de objetivos e de conflitos, os trade-offs da estratégia; e iv) considerar aspectos da cultura organizacional. 0 uso de múltiplas variáveis na medição de desempenho ambiental remete à decisão multicriterial. Para French (1986), dificilmente em uma decisão multicriterial se tem um modelo claro e uniformemente estruturado. Discussões mais aprofundadas sobre a teoria da decisão com enfoque multicriterial são encontradas em French (1986).

Um desempenho exige um modelo para mensuração e comunicação, obtido por construção mental. A construção mais abstrata é o termo teórico, que aglutina aspectos de uma definição suficientemente ampla, estruturados em construtos e conceitos. Os construtos também são construções abstratas, deliberadamente criadas para atender a um propósito científico, porém já mais próximas da realidade. 0 conceito, por fim, ainda não é o fenômeno, mas já pode comunicar suas implicações. Suas dimensões são representáveis por valores numéricos, os indicadores, que podem ser combinados e resumidos quantitativamente em índices, segundo esquemas teóricos em hierarquia que auxiliam a representação da realidade intangivel (LAKATOS; MARCONI, 1991).

Até onde se pesquisou, não foram encontradas referências que abordem especificamente o desempenho em DfE ou ecodesign ou, ao menos, a descrição da presença dessas práticas em um ambiente de projeto de produto.

A estrutura de desempenho, no caso o desempenho em DfE, foi organizada em formato de árvore (treelike). 0 formato de árvore, ou arborescência, é um grafo conexo, ou seja, todo vértice pode ser alcançado a partir de outro vértice qualquer (não há dois ou mais subconjuntos isolados no grafo), e sem ciclos internos, ou seja, não existe caminho interno que permita sair e um ponto e retornar a este ponto seguindo sempre em frente (BOAVENTURA NETO; JURKIEWICZ, 2009). Uma arborescência usada em problemas de decisão é como ilustrada na Figura 1 e seus ramos podem ser ponderados por métodos de apoio à decisão multicriterial, tal como o AHP (Analytic Hierarchy Process). Como exemplo, pode-se observar em Madu, Kuei e Madu (2002) um caso de aplicação da AHP na integração dos objetivos ambientais em ecodesign.

Segundo Forman e Selly (2001), o AHP obriga os decisores a considerar percepções, experiências, intuições e incertezas de modo racional, gerando escalas de prioridade ou pesos. É uma metodologia de decisão compensatória porque alternativas frágeis para um objetivo podem ter desempenho forte em outros objetivos. 0 AHP opera em três passos: i) descrição da situação complexa de interesse sob a forma de hierarquias conceituais, formadas por critérios e subcritérios até que, segundo os decisores, o enunciado do problema tenha sido suficientemente descrito; ii) comparação, duas a duas, das influências dos critérios e subcritérios nos entes superiores da hierarquia; e iii) computação dos resultados.

A importância relativa dos critérios surge por comparações pareadas. Para um nível com $n$ critérios, são requeridas n. $(n-1) / 2$ comparações entre $a_{i} e$ $\mathrm{a}_{\mathrm{j}}, \forall \mathrm{i}, \mathrm{j}$, construindo-se uma matriz de preferências $C_{i, j}(n \times n)$ (SAATY, 1991). A comparação é feita respondendo-se às questões:

- Ao influenciar o nível acima, o critério $a_{i}$ é mais importante, menos importante ou igual em importância ao critério $a_{j}, \forall i, j$; e;

- Para os $\mathrm{a}_{\mathrm{i}, \mathrm{j}}$ não equivalentes, e dado que $\mathrm{a}_{\mathrm{i}}$ é mais importante do que $a_{i}, a_{i}$ é: i) um pouco mais importante do que $\mathrm{a}_{\mathrm{j}}$; ii) muito mais importante do que $a_{j}$; iii) muito fortemente mais importante do que $a_{j}$; ou iv) absolutamente mais importante do que $a_{j}$.

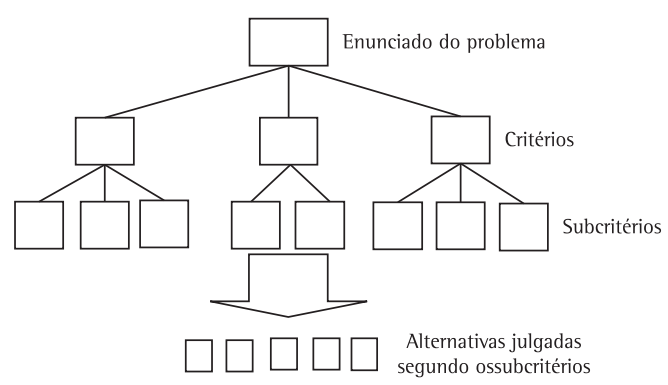

Figura 1. Estrutura de decisão hierárquica. Fonte: adaptado de Forman e Selly (2001). 
A matriz de preferências é preenchida segundo a Tabela 1. Valores intermediários se destinam a situações intermediárias, nas quais foi necessário discernir mais claramente entre duas alternativas muito parecidas, segundo o critério de julgamento. Por fim, calcula-se a importância relativa dos critérios, encontrando-se os autovetores com máximos autovalores da matriz. Os componentes do autovetor são as prioridades dos critérios e o autovalor dá a medida de consistência do julgamento, dentro das bases propostas pelo método. Se A for a matriz de comparações, deve-se encontrar o vetor de prioridades $\mathrm{w}$, tal que satisfaça a Equação 1. A razão de consistência CR é calculada pela Equação 2.

$$
\begin{aligned}
& \text { A.w }=\lambda_{\text {max }} \cdot w \\
& C R=\left[\lambda_{\text {max }}-n\right] /[\operatorname{RR} .(n-1)]
\end{aligned}
$$

na qual: i) $n$ é o número de critérios; ii) $\lambda_{\max } \leq n$ é o maior autovalor; e iii) IR é o índice randômico médio, extraído de tabela obtida por simulações com amostras de matrizes n-dimensionais. A Tabela 1 surge em Saaty (1991, p. 27). Por exemplo, se um decisor considera $a_{1}$ uma vez e meia mais importante que $\mathrm{a}_{2}$ e $\mathrm{a}_{2}$ duas vezes mais importante que $a_{3}$, este decisor deve considerar $a_{1}$ três vezes mais importante que $a_{3}$. Se for outro o julgamento, há inconsistência, medida pela CR. A CR é a probabilidade de que os valores da matriz sejam puramente aleatórios e, portanto, não constituam uma estrutura de preferências originada de um julgamento racional. Saaty (1991) sugere arbitrariamente que se aceite $C R<0,10$, do contrário recomenda a identificação e reformulação dos julgamentos que contribuíram para a inconsistência. Hogart (1988), no entanto, alerta que se deve contar com certa inconsistência no modelo mental e em julgamentos de decisores, o que pode ser apreendido pela CR.

A fim de manter a coerência de terminologia utilizada neste trabalho, adotam-se: DfE ou ecodesign é o termo de topo; as práticas do DfE são os construtos e os elementos que compõem as práticas do DfE, são os itens de aplicação (também denominados conceitos).

\begin{tabular}{|c|c|c|c|}
\hline $\begin{array}{c}\text { Se } a_{i} \text { em relação } \\
a a_{j}=\end{array}$ & $\begin{array}{c}\text { Então } \\
c_{\mathrm{ij}}=\end{array}$ & $\begin{array}{c}\text { Se } a_{i} \text { em relação } \\
a a_{j}=\end{array}$ & $\begin{array}{c}\text { Então } \\
c_{\mathrm{ij}}=\end{array}$ \\
\hline lgual & 1 & lgual & 1 \\
\hline $\begin{array}{l}\text { Um pouco mais } \\
\text { importante }\end{array}$ & 3 & $\begin{array}{l}\text { Um pouco menos } \\
\text { importante }\end{array}$ & $1 / 3$ \\
\hline $\begin{array}{l}\text { Muito mais } \\
\text { importante }\end{array}$ & 5 & $\begin{array}{l}\text { Muito menos } \\
\text { importante }\end{array}$ & $1 / 5$ \\
\hline $\begin{array}{l}\text { Fortemente mais } \\
\text { importante }\end{array}$ & 7 & $\begin{array}{l}\text { Fortemente menos } \\
\text { importante }\end{array}$ & $1 / 7$ \\
\hline $\begin{array}{l}\text { Absolutamente } \\
\text { mais importante }\end{array}$ & 9 & $\begin{array}{l}\text { Absolutamente } \\
\text { menos importante }\end{array}$ & $1 / 9$ \\
\hline
\end{tabular}

Tabela 1. Opções de preferência com base em comparação pareada.

\section{Delineamento da pesquisa}

Sellitto e Ribeiro (2004) propuseram uma estrutura para medição de objetos intangíveis complexos, baseada na teoria das medições e na filosofia da ciência, que pode ser usada para medição de desempenho ambiental. 0 ente de topo da estrutura é o termo teórico, explicado por construtos latentes, que se sustentam em conceitos, mais próximos da realidade, explicados por indicadores. Construtos e conceitos estruturam a grandeza intangivel em níveis hierárquicos, construídos por teoria, conhecimento empírico ou opinião de especialistas. A medição assume a forma de sistema, desdobrada em subsistemas que recompõem e medem o sistema como um todo, garantindo consistência e confiabilidade na medição.

Uma parte da pesquisa teve como método a modelagem. Neste método, são usadas técnicas matemáticas para descrever o funcionamento ou as relações observadas no objeto de estudo (FILIPPINI, 1997).

0 objetivo principal deste trabalho é avaliar o desempenho do DfE em uma empresa. Para que o objetivo principal seja atingido, os seguintes objetivos secundários foram estabelecidos: i) identificar construtos latentes que expliquem o DfE; ii) propor um modelo, suportado por método de decisão multicriterial, para avaliar a importância relativa de cada construto do DfE; iii) elaborar um instrumento de avaliação da presença ou grau de aplicação de cada construto do DfE; e iv) aplicar o método proposto em um caso e analisar os resultados. As premissas consideradas para a proposição do método seguiram o exposto em Sellitto, Borchardt e Pereira (2010), com exceção da primeira; para o caso deste trabalho, assume-se que DfE pode ser desdobrado em construtos latentes formados a partir dos itens de aplicação.

As etapas de pesquisa foram: i) construção de uma estrutura arborescente capaz de representar o termo de topo DfE e seus construtos latentes, baseada no referencial teórico; ii) ponderação, válida apenas para o caso, da estrutura pela AHP; iii) desdobramento dos construtos em itens de aplicação do DfE, baseado no referencial teórico, e elaboração de instrumento de avaliação (questionário) para identificar o grau de presença de cada item no caso; e iv) aplicação do questionário e uso dos resultados em eventuais reformulações estratégicas.

A partir do referencial teórico, identificaram-se os construtos latentes que compõem e explicam as práticas do DfE (Quadro 2). Em sessões de grupo focado com especialistas da indústria moveleira e da indústria química e com os pesquisadores, os construtos foram discutidos e o grupo concluiu que são aplicáveis para a empresa em estudo, não havendo a necessidade de exclusões ou inclusões de novos 
construtos. Dessa forma, foi estabelecida a estrutura arborescente capaz de representar o termo de topo DfE através dos construtos latentes. Os procedimentos do grupo focado seguiram o proposto em Ribeiro e Newmann (2003), Hesse-Biber e Leavy (2005).

Para avaliar a importância relativa de cada construto do DfE, usou-se a AHP e os critérios da Tabela 1. A sessão de ponderação das importâncias dos construtos foi mediada por pesquisador e contou com a presença de mais três pesquisadores e quatro gestores da empresa que atuam em desenvolvimento de produtos, gestão ambiental, gestão da qualidade e produção. Como resultado, obteve-se um vetor representando a prioridade (ou peso ou ainda importância relativa) de cada construto do DfE válido para o caso. Após a aplicação da AHP, os resultados foram discutidos e, de acordo com a percepção dos gestores, refletem a estratégia da empresa no que se refere às prioridades e alocação de recursos para cada construto. 0 grau de importância de cada construto é expresso em percentual e a soma de todos os graus é de 100\%.

A próxima etapa consistiu do desdobramento dos construtos em itens de aplicação do DfE. Foi proposto ao grupo de foco um desdobramento inicial, construído a partir do referencial teórico (Quadro 2). Após discussões, foi elaborado um instrumento com 32 quesitos, que foi testado pelo grupo de pesquisadores em simulações internas e aplicação com dois praticantes da primeira etapa. Os resultados das simulações e dos testes motivaram mudanças no teor e no formato das perguntas, chegando-se ao formato final do questionário. Para as respostas, atribuiu-se uma escala com valores de 1 a 5 [ 1 = quesito nunca presente; 5 = quesito sempre presente] ou NA (quesito não aplicável) para cada questão. 0 questionário foi respondido pelos quatro gestores que participaram da avaliação da importância dos construtos. Cada uma das respostas era discutida e o valor final atribuído para cada questão foi consensado.
A análise das respostas atribuídas a cada questão permitiu obter um índice percentual de quanto o DfE está presente nas atuais práticas de desenvolvimento de produto da empresa. Ao final, obtêm-se a importância relativa de cada construto e o grau de presença de cada um deles na empresa; o valor final é formado por parcelas que podem ser usadas em uma eventual reformulação da estratégia de projeto da empresa, caso esta decida aumentar a presença do DfE em suas práticas.

A empresa em que foi testada a avaliação de desempenho do DfE tem seis unidades fabris no Brasil; o estudo ocorreu em planta situada na região sul do Brasil, considerada de grande porte. Os principais produtos fabricados nesta planta são adesivos e laminados para a indústria calçadista, moveleira e automotiva. Por parte da empresa, participaram da pesquisa quatro gestores. Todos têm autonomia na gestão, dominam plenamente os processos produtivos, as aplicações dos produtos e os processos logísticos associados a estes. A empresa já emitiu diretriz de projeto estimulando seus praticantes a adotarem práticas de DfE. No entanto, talvez por falta de base conceitual, a empresa ainda não emitiu um programa formal elaborado de adoção de DfE. Em função das iniciativas observadas e projetos focados nas questões ambientais e considerando a experiência dos pesquisadores e gestores que participaram da pesquisa, identificou-se na empresa o potencial e as condições propostas por Vercalsteren (2001) e Reyes, Millet e Bissaud (2006) para o DfE.

\section{Aplicação do método proposto e resultados}

\subsection{Construção da estrutura arborescente do DfE}

A construção da estrutura arborescente seguiu o descrito no capítulo Delineamento da pesquisa. A Figura 2 apresenta a estrutura arborescente definida pelo grupo de foco, usada para explicar o DfE.

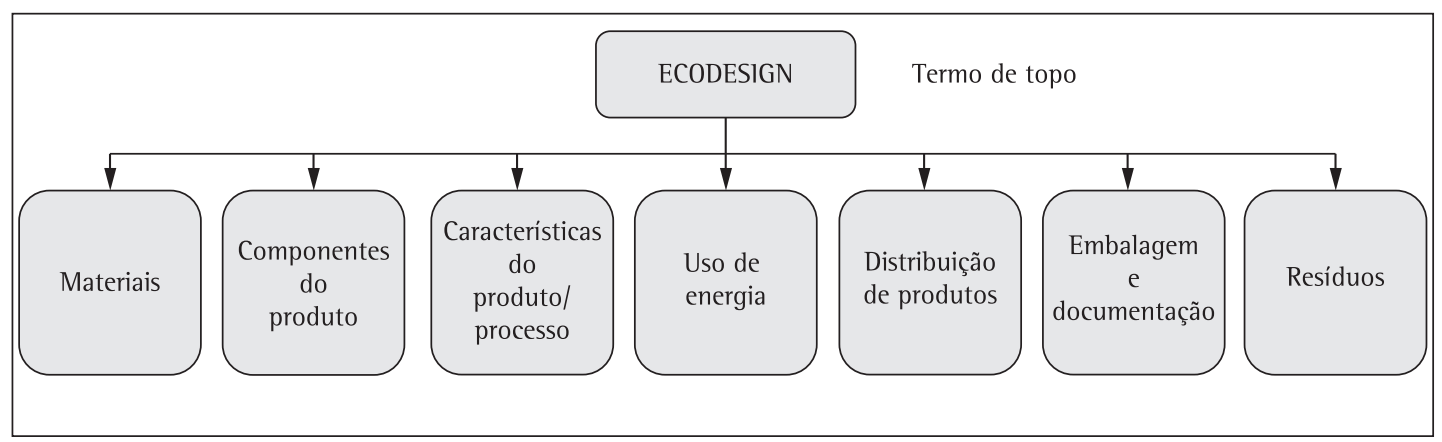

Figura 2. Estrutura arborescente representativa do DfE. 


\subsection{Ponderação da estrutura pela AHP para obtenção do grau de importância dos construtos}

Os resultados dos julgamentos dos membros do grupo usando a AHP e o respectivo vetor de prioridades surgem na Tabela 2. Obteve-se: materiais com 12\% de importância relativa; componentes do produto com 3\%; características do produto e do processo com 34\%; uso de energia com 3\%; distribuição de produtos com 8\%; embalagem e documentação com $11 \%$ e resíduos com $29 \%$ de importância relativa para o DfE. A CR foi de 6,4\% $(<10 \%)$, o que indica que as preferências dos decisores têm racionalidade aceitável, segundo os proponentes do método.

\subsection{Aplicação do questionário}

A Tabela 3 apresenta as respostas dos gestores, obtida por consenso.

\subsection{Grau de presença de DfE}

A Tabela 4 apresenta os resultados compilados. Nas duas primeiras colunas, tem-se os construtos e as prioridades ou importâncias. Na terceira, tem-se os graus de presença do DfE nos construtos, obtidos pela média das respostas dos quesitos do construto, em percentual da prioridade e em valores absolutos ( $\mathrm{pp}=$ pontos percentuais). Por exemplo, para o primeiro construto, materiais, a prioridade é $12 \%$ e a presença é de $60 \%$ da prioridade; $60 \%$ de $12 \%$ correspondem a 7,2 pp. Na última coluna, tem-se as lacunas, ou seja, o quanto o construto deixou de contribuir para máxima pontuação. As lacunas são úteis caso se decida modificar a estratégia de projeto, rearranjando recursos para aumentar a presença do DfE nas práticas de desenvolvimento de produto.

\section{Discussão}

0 construto Características do produto/processo é o mais prioritário e é atendido em 85\%. 0 segundo é Resíduos, atendido em 64,1\%. 0 terceiro é Materiais, atendido em $60 \%$. Embalagem e documentação é o quarto, atendido em 51,8\%. Seguem Distribuição de produtos/estoques, Uso de energia e Componentes do produto, atendidos em 50\%, 53,3\% e 33,3\%. Globalmente, a empresa pratica $67 \%$ do que poderia praticar em suas práticas de DfE.

Para os gestores, as importâncias relativas dos construtos do DfE refletem a estratégia e as ações da empresa. Foca-se primordialmente nas características do produto e processo, vinculado diretamente com a gestão dos resíduos. As características inovadoras dos produtos fazem a diferenciação dos produtos. Prioriza-se a não geração dos resíduos; não sendo possivel, busca-se a maximização do uso dos resíduos nos próprios processos produtivos da empresa. Energia foi um dos construtos com menor grau de importância; o consumo e os respectivos custos com energia na empresa são considerados baixos comparativamente aos demais; ainda assim, há uma preocupação para que o usuário dos produtos da empresa (tipicamente montadoras de calçados e empresas moveleiras) reduza o consumo de energia na cura e secagem dos adesivos, focando-se dessa maneira na redução do consumo durante o uso do produto. Componentes também apresenta baixa importância relativa; observa-se a pouca presença desse construto devido às características técnicas dos produtos. A Figura 3 apresenta graficamente os valores.

Caso se deseje simplesmente aumentar a presença total do DfE, as lacunas apontadas devem ser reduzidas por ações gerenciais que podem demandar novos recursos. Caso se considere que recursos são finitos e geralmente restringidos, antes de alocar mais recursos ao desenvolvimento de novos produtos, talvez seja melhor realocar os atuais.

Tabela 2. Matriz de preferência dos construtos do DfE.

\begin{tabular}{|c|c|c|c|c|c|c|c|c|}
\hline & 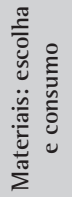 & 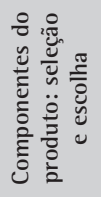 & 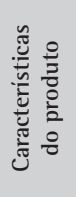 & 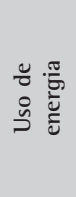 & 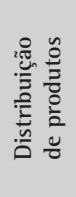 & 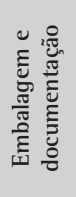 & $\frac{\tilde{o}}{\stackrel{n}{0}}$ & $\frac{\frac{0}{0}}{\frac{\pi}{0}} \frac{0}{\frac{0}{0}}$ \\
\hline Materiais: escolha e consumo & 1 & 3 & $1 / 3$ & 5 & 3 & 1 & $1 / 3$ & 12 \\
\hline Componentes do produto: seleção e escolha & $1 / 3$ & 1 & $1 / 7$ & 1 & $1 / 3$ & $1 / 5$ & $1 / 9$ & 3 \\
\hline Características do produto & 3 & 7 & 1 & 7 & 3 & 3 & 3 & 34 \\
\hline Uso de energia & $1 / 5$ & 1 & $1 / 7$ & 1 & $1 / 5$ & $1 / 7$ & $1 / 9$ & 3 \\
\hline Distribuição de produtos & $1 / 3$ & 3 & $1 / 3$ & 5 & 1 & 1 & $1 / 5$ & 8 \\
\hline Embalagem e documentação & 1 & 5 & $1 / 3$ & 7 & 1 & 1 & $1 / 5$ & 11 \\
\hline Resíduos & 3 & 9 & $1 / 3$ & 9 & 5 & 5 & 1 & 29 \\
\hline
\end{tabular}


Tabela 3. Aplicação dos quesitos referentes aos construtos do DfE.

Construto

1) Capacidade de usar matéria-prima mais próxima de seu estado natural

2) Capacidade de evitar misturas de materiais não compatíveis visando posterior reciclagem, reuso ou reaproveitamento dos materiais

Materiais 3) Capacidade de eliminar ou não usar substâncias tóxicas, perigosas ou carcinogênicas

4) Capacidade de eliminar ou não usar matérias-primas que geram resíduos classe 1 - perigosos

5) Capacidade de usar materiais reciclados e/ou renováveis

6) Capacidade de limitar as emissões atmosféricas originadas pelo emprego de compostos orgânicos voláteis 2

\begin{tabular}{|c|c|c|}
\hline & 6) Capacidade de limitar as emissões atmosféricas originadas pelo emprego de compostos orgânicos voláteis & 2 \\
\hline \multirow{4}{*}{$\begin{array}{l}\text { Componentes } \\
\text { do produto }\end{array}$} & 7) Capacidade de recuperar componentes ou usar componentes recuperados & 2 \\
\hline & 8) Capacidade de facilitar o acesso aos componentes & NA \\
\hline & 9) Capacidade de identificar materiais e componentes para viabilizar posterior reciclagem ou reaproveitamento & 2 \\
\hline & 10) Capacidade de determinar o grau de reciclagem do material e componente & 1 \\
\hline \multirow{4}{*}{$\begin{array}{c}\text { Características } \\
\text { do produto/ } \\
\text { processo }\end{array}$} & 11) Capacidade de elaborar produtos com formas mais simples e que reduzam o uso ou consumo de matérias-primas & 4 \\
\hline & 12) Capacidade de projetar produtos com vida útil maior & 5 \\
\hline & 13) Capacidade de projetar produtos multifuncionais & 4 \\
\hline & 14) Capacidade de realizar upgrade no produto & 4 \\
\hline \multirow{4}{*}{$\begin{array}{l}\text { Uso de } \\
\text { energia }\end{array}$} & 15) Capacidade de usar energia proveniente de recursos renováveis & 2 \\
\hline & 16) Capacidade de empregar dispositivos de redução de consumo de energia durante o uso do produto & 3 \\
\hline & 17) Capacidade de reduzir consumo de energia durante a produção do produto & 3 \\
\hline & 18) Capacidade de reduzir consumo de energia durante o armazenamento do produto & NA \\
\hline \multirow{4}{*}{$\begin{array}{l}\text { Distribuição } \\
\text { de produtos/ } \\
\text { estoques }\end{array}$} & 19) Capacidade de planejar racionalmente e otimizar a logística de distribuição & 5 \\
\hline & 20) Capacidade de privilegiar fornecedores e distribuidores localizados mais próximos & 1 \\
\hline & $\begin{array}{l}\text { 21) Capacidade de minimizar os estoques de matérias-primas, durante o processo produtivo, de produto acabado } \\
\text { e de produto para reuso }\end{array}$ & 3 \\
\hline & 22) Capacidade de usar modal de transporte mais eficiente em termos energéticos & 1 \\
\hline \multirow{5}{*}{$\begin{array}{l}\text { Embalagem e } \\
\text { documentação }\end{array}$} & 23) Capacidade de reduzir peso e complexidade das embalagens & 2 \\
\hline & 24) Capacidade de usar embalagens que possam ser reaproveitadas & 2 \\
\hline & $\begin{array}{l}\text { 25) Capacidade de usar embalagens produzidas a partir de matérias-primas reaproveitadas (ex.: reciclados como } \\
\text { papel, termoplásticos) }\end{array}$ & 3 \\
\hline & 26) Capacidade de usar documentação eletrônica & 3 \\
\hline & 27) Capacidade de usar produtos com refil & 3 \\
\hline \multirow{5}{*}{ Resíduos } & 28) Capacidade de minimizar os resíduos gerados no processo produtivo & 4 \\
\hline & 29) Capacidade de minimizar os resíduos gerados durante o uso do produto & 3 \\
\hline & 30) Capacidade de reaproveitar os resíduos gerados & 4 \\
\hline & 31) Capacidade de garantir limites aceitáveis de emissões & 3 \\
\hline & 32) Capacidade de eliminar a presença de resíduos classe 1 - perigosos & 2 \\
\hline
\end{tabular}

Tabela 4. Grau de presença do DfE.

\begin{tabular}{cccc}
\hline Construto & Prioridades (\%) & Grau de presença & Lacuna \\
\hline Materiais & 12 & $60 \%(7,2 \mathrm{pp})$ & $4,8 \mathrm{pp}$ \\
Componentes do produto & 3 & $33,3 \%(1,0 \mathrm{pp})$ & $2,0 \mathrm{pp}$ \\
Características do produto/processo & 34 & $85 \%(28,9 \mathrm{pp})$ & $5,1 \mathrm{pp}$ \\
Uso de energia & 3 & $53,33 \%(1,6 \mathrm{pp})$ & $1,4 \mathrm{pp}$ \\
Distribuição de produtos/estoques & 8 & $50 \%(4,0 \mathrm{pp})$ & $4,0 \mathrm{pp}$ \\
Embalagem e documentação & 11 & $51,8 \%(5,7 \mathrm{pp})$ & $5,3 \mathrm{pp}$ \\
Resíduos & 29 & $64,1 \%(18,6 \mathrm{pp})$ & $10,4 \mathrm{pp}$ \\
Total & 100 & $67,0 \mathrm{pp}$ & \\
\hline
\end{tabular}

Para tal realocação, pode-se usar a análise gráfica bidimensional [lmportância - Desempenho]. Até quanto se pesquisou, a referência mais antiga ao método foi Slack (1993), seguindo-se Paiva, Carvalho e Fenstenseifer (2004) e Carvalho e Laurindo (2007). Seu fundamento é que um fator de sucesso (construto) deve ter desempenho (presença) avaliado próximo à importância atribuída. Segundo esse fundamento, em um gráfico [Importância - Presença], o ideal seria que os construtos se distribuíssem ao longo de uma diagonal: construtos de mais importância teriam mais presença. Caso um construto tenha muito mais 


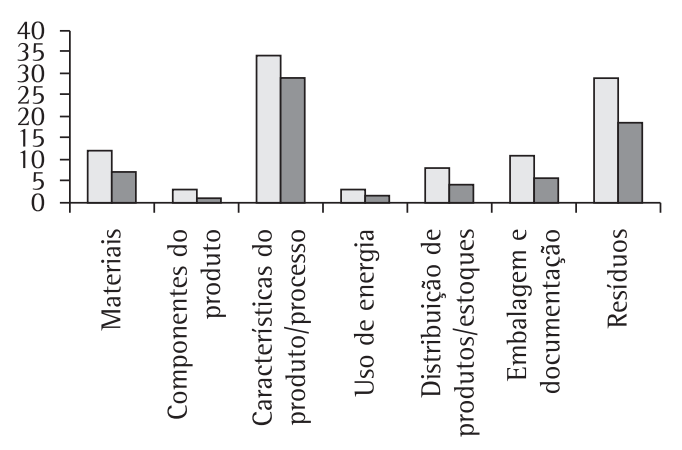

$\square$ Importância (\%)

$\square$ Aplicação (em pontos percentuais)

Figura 3. Importância e aplicação dos construtos do DfE.

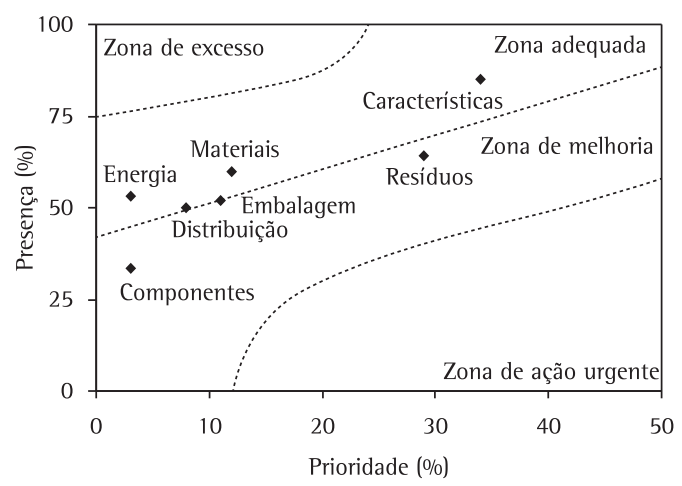

Figura 4. Análise [Prioridade $\times$ Presença] dos construtos do DfE.

importância do que presença, a atual estratégia o valoriza pouco. Para reposicionar o construto, uma alternativa é alocar novos recursos. Outra é realocar recursos empregados nos construtos de mais presença do que importância, se houver.

A Figura 4 apresenta a análise. 0 espaço bidimensional é composto pela zona de ação urgente (prioridade muito maior que presença); zona de melhoria (prioridade maior que presença); zona adequada (prioridade e presença equilibradas); e zona de excesso (prioridade menor que presença). Características, Materiais e Energia são construtos equilibrados e não devem ser modificados. Componentes e Resíduos devem ser alvo de ações de melhoria. Distribuição e Embalagem estão em região limítrofes às zonas. Não há construtos em zona urgente nem em zona de excesso, portanto, em princípio, não há construtos dos quais retirar recursos produtivos para alocação a outros construtos.
Uma crítica que pode ser feita ao método diz respeito à subjetividade do grupo focado. Para evitar que o grupo tenda a favorecer determinados construtos, este pode ser composto por especialistas ad hoc, sem interesse na operação, mas que venham a conhecê-la, para não deixar de apontar os aspectos que a empresa pode e deve alavancar em DfE. Outra crítica diz respeito ao método AHP, que traz em seu bojo uma parcela de inconsistência e tem sofrido críticas acerca de sua resposta quando há entradas ou saídas de critérios. 0 uso do AHP foi uma delimitação do projeto de pesquisa no qual o caso se insere. Caso essa crítica seja insuperável, a contribuição essencial do caso passa a ser o método e não o resultado específico do caso.

Apesar das delimitações descritas, a análise dos construtos do DfE considerando a importância atribuída pela empresa para cada construto e o seu grau de presença, corroborado pela interpretação da Figura 4, efetivamente indica coerência por parte da empresa na alocação dos recursos: as maiores prioridades têm os maiores graus de presença sem, contudo, estarem na considerada zona de excesso.

\section{Conclusões}

0 objetivo principal deste trabalho foi avaliar o desempenho do DfE em uma empresa da indústria química. Os construtos que compõem e explicam o DfE foram estabelecidos no referencial teórico, partindo do conjunto de práticas propostas por Fiksel (1996), Wimmer et al. (2005) e Luttropp e Lagersted (2006). 0 grupo de gestores que participou da pesquisa entendeu que sua abrangência é adequada, não sendo necessário incluir outras práticas. A importância dos construtos foi obtida a partir de uma estrutura arborescente representativa do DfE ponderada por meio da AHP. Um instrumento de avaliação foi utilizado para verificar o grau de presença de cada construto. Por fim, determinou-se o desempenho global da empresa em relação às práticas do DfE.

Entende-se que este trabalho contribui para o posterior desenvolvimento de indicadores de desempenho associados ao DfE, complementando o exposto por Cerdan et al. (2009), Cabezas et al. (2005), Svensson et al. (2006) e Daub (2007). Os principais indicadores de desempenho poderiam estar relacionados com os itens do DfE inseridos nas práticas com maior grau de importância.

Os achados de pesquisa, sob a ótica da empresa estudada, possibilitarão que a tomada de decisão, envolvendo recursos e prioridade para ações relacionadas ao DfE, seja focada nos construtos que mais contribuem. Sob a ótica científica, a contribuição 
reside na elaboração de um método aplicável a outras organizações, inclusive de outras indústrias. Entende-se que o estudo poderá ser continuado, avaliando-se o desempenho de uma indústria de forma ampla ou, ainda, estabelecendo indicadores de desempenho atrelados a cada construto.

Os resultados apresentados neste artigo são decorrentes das pesquisas efetuadas dentro do contexto do projeto "Diretrizes para gestão de projetos de produto e operações de manufatura e assistência técnica orientada para o ecodesign". Este projeto tem apoio do CNPq.

\section{Referências}

AMMENBERG, J.; HJELM, 0.; QUOTES, P. The Connection between Environmental Management Systems and Continual Environmental Performance Improvements. Corporate Environmental Strategy, v. 9, n. 2, p. 183-192, 2002.

BEITZ, W. Designing for Ease of Recycling. Journal of Engineering Design, v. 4, n. 1, p. 11-23, 1993. http:// dx.doi.org/10.1080/09544829308914769

BHAMRA, T. Ecodesign: the search for new strategies in product development. Journal of Engineering Manufacture, v. 218, n. 5, p. 557-569, 2004. http:// dx.doi.org/10.1177/095440540421800509

BITITCl, U. Modelling of performance measurement systems in manufacturing enterprises. International Journal of Production Economics, v. 42, p. 137-147, 1995. http:// dx.doi.org/10.1016/0925-5273(95)00172-7

BOAVENTURA NETO, P.; JURKIEWICZ, S. Grafos: introdução e prática. São Paulo: Blucher, 2009.

BORCHARDT, M. et al. Considerações sobre ecodesign: um estudo de caso na indústria eletrônica automotiva. Ambiente \& Sociedade, v. 11, n. 2, p. 1-13, 2008.

BORCHARDT, M. et al. Adopting ecodesign practices: case study of a midsized automotive supplier. Environmental Quality Management, v. 19, p. 7-22, 2009. http://dx.doi. org/10.1002/tqem.20232

BORCHARDT, M. et al. Reprojeto do contraforte: um caso de aplicação do ecodesign em uma empresa do setor calçadista. Revista Produção, v. 20, n. 3, p. 392-403, 2010.

BREZET, $\mathrm{H}$. Dynamics in ecodesign practice. Industry and Environment, v. 20, n. 1-2, p. 21-25, 1997.

CABEZAS, $\mathrm{H}$. et al. Sustainable systems theory: ecological and other aspects. Journal of Cleaner Production, v. 13, n. 5, p. 455-467, 2005. http://dx.doi.org/10.1016/j. jclepro.2003.09.011

CARVALHO, M; LAURINDO, F. Estratégia competitiva: dos conceitos à implementação. São Paulo: Ed. Atlas, 2007.

CERDAN, C. et al. Proposal for new quantitative ecodesign indicators: a first case study. Journal of Cleaner Production, v. 17, n. 18, p. 1638-1643, 2009. http:// dx.doi.org/10.1016/j.jclepro.2009.07.010

CHARTER, M. Managing the eco-design process. Journal of Sustainable Product Design, v. 2, p. 48-51, 1997.

COSTA, M. M. Princípios de Ecologia Industrial Aplicados à Sustentabilidade Ambiental e aos Sistemas de Produção de Aço. 2002. Tese (Doutorado em Engenharia)Universidade Federal do Rio de Janeiro, Rio de Janeiro, 2002.

DAUB, C. Assessing the quality of sustainability reporting: an alternative methodological approach. Journal of Cleaner Production, v. 15, n. 1, p. 75-85, 2007. http://dx.doi. org/10.1016/j.jclepro.2005.08.013

FIKSEL, J. Design for Environment. New York: Mc Graw Hill, 1996.

FILIPPINI, R. Operations Management research: some reflections on evolution, models and empirical studies in OM. International Journal of Operations \& Production Management, v. 17, n. 7-8, p. 655-670, 1997. http:// dx.doi.org/10.1108/01443579710175583

FORMAN, E.; SELLY, M. Decisions by objectives. Expert Choice, Inc., 2001. Disponível em: <http://www.expertchoice.com>. Acesso em: maio 2004.

FRENCH, S. Decision Theory: an introduction to the mathematics of rationality. West Sussex: Ed. Ellis Horwood, 1986.

HARTSHORN, J. Toward sustainability: expanding the scope of environmental management system. In: KHARE, A. (Org.). Emerging dimensions of environmental sustainability: a canadian perspective of innovative practices. EdingenNeckarhausen: Fachbuch Verlag Winkler, 2005.

HESSE-BIBER, S.; LEAVY, P. The practice of qualitative research. Thousand Oaks: Ed. Sage, 2005.

HOGART, R. Judgement and choice. Essex: John Wiley \& Sons, 1988.

JIMÉNEZ, J.; LORENTE, J. Environmental performance as an operations objective. International Journal of Operations \& Production Management, v. 21, n. 12, p. 1553-1572, 2001. http://dx.doi. org/10.1108/01443570110410900

KARLSSON, R.; LUTTROPP, C. Ecodesign: What's happening? An overview of the subject area of ecodesign and the papers in this Special Issue. Journal of Cleaner Production, v. 14, n. 15-16, p. 1291-1298, 2006. http:// dx.doi.org/10.1016/j.jclepro.2005.11.010

KAZAZIAN, T. Haverá a ldade das Coisas Leves - Design e Desenvolvimento Sustentável. São Paulo: Ed. SENAC, 2005.

KNIGHT, P.; JENKIS, J. Adopting and applying eco-design techniques a practioners perspective. Journal of Cleaner Production, v. 17, n. 5, p. 549-558, 2009. http://dx.doi. org/10.1016/j.jclepro.2008.10.002

LAKATOS, E.; MARCONI, M. Metodologia científica. São Paulo: Ed. Atlas, 1991.

LEE, S.; KLASSEN, R. Drivers and enablers that foster environmental management capabilities in Small and Medium Sized suppliers in supply chains. Production and Operations Management, v. 17, n. 6, p. 573-586, 2008. http://dx.doi.org/10.3401/poms.1080.0063

LINDAHL, M. Engineering designers' experience of design for environment methods and tools: requirement definitions from an interview study. Journal of Cleaner Production, v. 14, n. 5, p. 487-496, 2006. http://dx.doi.org/10.1016/j. jclepro.2005.02.003

LUTTROPP, C.; LAGERSTEDT, J. Ecodesign and the ten golden rules: generic advice for merging environmental aspects into product development. Journal of Cleaner 
Production, v. 14, n. 15-16, p.1396-1408, 2006. http:// dx.doi.org/10.1016/j.jclepro.2005.11.022

MADU, C.; KUEl, C.; MADU, 1. A hierarchic metric approach for integration of green issues in manufacturing: a paper recycling application. Journal of Environmental Management, v. 64, p. 261-272, 2002. PMid:12040959. http://dx.doi.org/10.1006/jema.2001.0498

MELO, J.; PEGADO, C. 2006. Ecoblock: A method for integrated environmental performance evaluation of companies and products (construction case-study). Disponivel em <http://gasa.dcea.fct.unl.pt/ecoblock/ EcoBalance_02.pdf>. Acesso em: 03 maio 2006.

PARK, P.; TAHARA, K. Quantifying producer and consumerbased eco-efficiencies for the identification of key ecodesign issues. Journal of Cleaner Production, v. 16, p. 95-104, 2008. http://dx.doi.org/10.1016/j. jclepro.2006.11.003

PAIVA, E.; CARVALHO, L; FENSTENSEIFER, J. Estratégia de produção e de operações. Porto Alegre: Ed. Bookman, 2004.

PEGADO, C.; MELO J.; RAMOS, T. Ecoblock: Método de avaliação do desempenho ambiental, 2001. Disponível em: <http://gasa.dcea.fct.unl.pt/ecoblock/Apea_01. pdf>. Acesso em: 03 maio 2006.

REYES, T.; MILLET, S; BISSAUD, D. Study of Ecodesign integration process in French companies. In: CIRP INTERNATIONAL CONFERENCE ON LIFE CYCLE ENGINEERING, 13., 2006, Belgium. Proceedings... Belgium, 2006.

RIBEIRO, J.; NEWMANN, C. Planejamento e condução de grupos focados. In: RIBEIRO, J. (Org.). Grupos focados: teoria e aplicações. Porto Alegre: Ed. FEENG-UFRGSPPGEP, 2003.

SAATY, T. Método de Análise Hierárquica. São Paulo: Ed. Makron Books, 1991.

SELLITTO, M.; RIBEIRO, J. Construção de indicadores para avaliação de conceitos intangíveis em sistemas produtivos. Gestão \& Produção, v. 11, n. 1, p. 75-90, 2004. http://dx.doi.org/10.1590/S0104-530X2004000100007

SELLITTO, M.; BORCHARDT, M.; PEREIRA, G. Avaliação multicriterial de desempenho: um estudo de caso na indústria de transporte coletivo de passageiros. Gestão \& Produção, v. 13, n. 2, p. 339-352, 2006. http://dx.doi. org/10.1590/S0104-530X2006000200014

SELLITTO, M.; BORCHARDT, M.; PEREIRA, G. Modelagem para avaliação de desempenho ambiental em operações de manufatura. Gestão \& Produção, v. 17, n. 1, p. 95-109, 2010. http://dx.doi.org/10.1590/S0104530X2010000100008

SLACK, N. Vantagem competitiva em manufatura: atingindo competitividade nas operações industriais. São Paulo: Ed. Atlas, 1993.

SVENSSON, N. et al. Environmental relevance and use of energy indicators in environmental management and research. Journal of Cleaner Production, v. 14, n. 2, p. 134-145, 2006. http://dx.doi.org/10.1016/j. jclepro.2005.01.004

TINGSTRÖM, J.; KARLSSON, R. The relationship between environmental analyses and the dialogue process in product development. Journal of Cleaner Production, v. 14, n. $15-16$, p. 1409-1419, 2006. http://dx.doi. org/10.1016/j.jclepro.2005.11.012

THORESEN, J. Environmental performance evaluation - a tool for industrial improvement. Journal of Cleaner Production, v. 7, n. 5, p. 365-370, 1999. http://dx.doi. org/10.1016/S0959-6526(99)00154-7

VERCALSTEREN, A. Integrating the ecodesign concept in small and medium-size enterprises: Experiences in the Flemish Region of Belgium. Environmental Management and Health, v. 12, p. 347-355, 2001. http://dx.doi. org/10.1108/EUM0000000005706

WIMMER, W. et al. Ecodesign in twelve steps: providing systematic guidance for considering environmental aspects and stakeholder requirements in product design and development. In: INTERNATIONAL CONFERENCE ON ENGINEERING DESIGN, 2005, Melbourne. Proceedings... Melbourne, 2005.

ZOBEL, T. et al. Identification and assessment of environmental aspects in an EMS context: an approach to a new reproducible method based on LCA methodology. Journal of Cleaner Production, v. 10, n. 4, p. 381-396, 2002. http://dx.doi.org/10.1016/S09596526(01)00054-3

\title{
Assessment of the presence of Design for Environment (DfE) practices in the product development of a chemical company
}

\begin{abstract}
This paper presents an assessment of the Design for Environment (DfE) or eco-design practices in the product development of a chemical company. To this end, the DfE was deployed in seven latent constructs, weighted by a multi-criteria method. The constructs were deployed in assessment items, resulting in a categorical questionnaire used to investigate how much each item is present in the company. Eventually, it was possible to identify the level of each construct and the global presence of DfE in the company. The company achieved 67\% of the maximum expected. The relative importance of each construct and the gaps between the importance and the application of each construct were also assessed. The most important construct was Characteristics of Product/Process and Wastes. This research allowed to identify the priorities of DfE application and may contribute to establish DfE performance references that can be long-term monitored.
\end{abstract}

\section{Keywords}

Design for Environment (DfE). Eco-design. Environment performance. Performance assessment. 